\title{
Nanocrystals-Related Synthesis, Assembly, and Energy Applications 2012
}

\author{
Bo Zou, ${ }^{1}$ William W. Yu, ${ }^{2}$ Jaetae Seo, ${ }^{3}$ Ting $\mathrm{Zhu}^{4}{ }^{4}$ and Michael Z. $\mathrm{Hu}^{5}$ \\ ${ }^{1}$ State Key Laboratory of Superhard Materials, Jilin University, Changchun 130012, China \\ ${ }^{2}$ College of Electronic Science and Engineering, Jilin University, Changchun 130012, China \\ ${ }^{3}$ Department of Physics, Hampton University, Hampton, VA 23668, USA \\ ${ }^{4}$ Philips Lumileds Lighting Company, San Jose, CA 95131, USA \\ ${ }^{5}$ Oak Ridge National Laboratory, Nuclear Science and Technology Division, Oak Ridge, TN 37831, USA
}

Correspondence should be addressed to Bo Zou, zoubo@jlu.edu.cn

Received 23 February 2012; Accepted 23 February 2012

Copyright (C) 2012 Bo Zou et al. This is an open access article distributed under the Creative Commons Attribution License, which permits unrestricted use, distribution, and reproduction in any medium, provided the original work is properly cited.

During the past decades, nanocrystals have attracted broad attention due to their unique shape- and size-dependent physical and chemical properties that differ drastically from their bulk counterparts. Hitherto, much effort has been dedicated to achieving rational controlling over the morphology, assembly, and related energy applications of the nanomaterials. Therefore, the ability to manipulate the morphology, size, and size distribution of inorganic nanomaterials is still an important goal in modern materials physics and chemistry. Especially, the world's demand for energy supply is causing a dramatic escalation of social and political unrest. Likewise, the environmental impact of the global climate change due to the combustion of fossil fuel is becoming increasingly alarming. These problems compel us to search for effective routes to build devices that can supply sustainable energy, with not only high efficiency but also environmental friendship. One of ways to relieve the energy crisis is to exploit devices based on renewable energy sources, such as solar energy and water power. Aiming at this exploration, the primary stage requires the design of appropriate strategies for the synthesis of high-quality nanocrystals with respect to size uniformity and superior electrochemical performances. As a consequence, we organize the current special issue for Journal of Nanomaterials to provide the authors with a platform and readers with the latest achievements of nanocrystals-related synthesis, assembly, and energy applications.
One of the papers presents the synthesis of single crystalline ZnTe hierarchical nanostructures by a simple thermal evaporation technology and discussed the corresponding growth mechanism. Another paper reports the synthesis of CdSe and CdTe quantum dots (QDs) from the bulk CdSe and CdTe material by evaporation/cocondensation using the solvated metal atom dispersion technique and refined digestive ripening. It is found that $\mathrm{PL}$ quantum yield of CdSe QDs and CdTe QDs upon covering with a $\mathrm{ZnS}$ shell was greatly enhanced. Another paper presents that nanosize TiB whiskers were successfully obtained by micropyretic synthesis which is considered as the one of the novel energy-saving processing techniques. The diffusioncontrolled reaction mechanism was proposed in this study.

One more paper presents the progress of $\mathrm{TiO}_{2}$ nanocrystals doped with rare earth ions in the aspect of nanooptic and photocatalytic properties. Another paper presents a search article in which $\mathrm{ZnO}$ nanoparticles were successfully synthesized at room temperature via mechanical milling, and the quantum size effect was interpreted in terms of absorption and PL spectra. An additional paper presents that $\mathrm{ZnO}$ micro-/nanocombs have been successfully synthesized on $\mathrm{Cu}$ substrate using a highly repeatable catalystfree chemical vapor deposition method. The cathodoluminescence spectrum was also measured on an individual $\mathrm{ZnO}$ comb and only a broad strong green emission band was observed. Another paper presents that mesoporous 
nanosheets of single-crystalline $\mathrm{Ni}(\mathrm{OH})_{2}$ were successfully prepared via a facile hydrothermal method. Furthermore, single-crystalline $\mathrm{NiO}$ mesoporous nanosheets were also obtained through a thermal decomposition of the obtained $\mathrm{Ni}(\mathrm{OH})_{2}$. Another paper presents that $\mathrm{BaSn}(\mathrm{OH})_{6}$ nanorods were synthesized via a novel two-step technique involving the preparation of precursor $\mathrm{Na}_{2} \mathrm{Sn}(\mathrm{OH})_{6}$ crystals in aqueous solution via hydrothermal method and the ion-exchange reaction between $\mathrm{Na}_{2} \mathrm{Sn}(\mathrm{OH})_{6}$ crystals and $\mathrm{Ba}^{2+}$ solution that followed, assisted by ultrasonic treatment. In addition, the powder $\mathrm{BaSnO}_{3}$ has been obtained by thermal treatment using $\mathrm{BaSn}(\mathrm{OH})_{6}$ nanorods as precursor. The ninth paper presents a microwave plasma synthesis of orthorhombic $\alpha$ $\mathrm{MoO}_{3}$ microplates with photoluminescence in a wavelength range of $430-440 \mathrm{~nm}$.

One more paper presents that various copper hierarchical structures assisted by surfactants were successfully fabricated combined with the electrodeposition technique and the possible formation mechanism of the copper hierarchical structures with various morphologies was discussed. Another paper presents that multicomponent Mg-based nano-quasicrystals were directly prepared through a simple route by using a water-cooled wedge-shaped copper mould.

An additional paper describes the research results on nanometers sizes subwavelength nanostructure fabrication by UV-curing of special nanocomposite material with selforganization and light self-focusing effects. Obtained results will be useful for diffraction limit overcoming in projection lithography as well as for deep lithography technique. Another paper presents that ionogels were prepared by ultrasonic-assisted sol-gel process and it has been found that ultrasonic irradiation affected the gelation dynamics and kinetics in pore parameters and vibrational properties of the IL.

\section{Acknowledgments}

The editors would like to thank the authors for their contributions to this special issue, the reviewers for their time and dedication. 

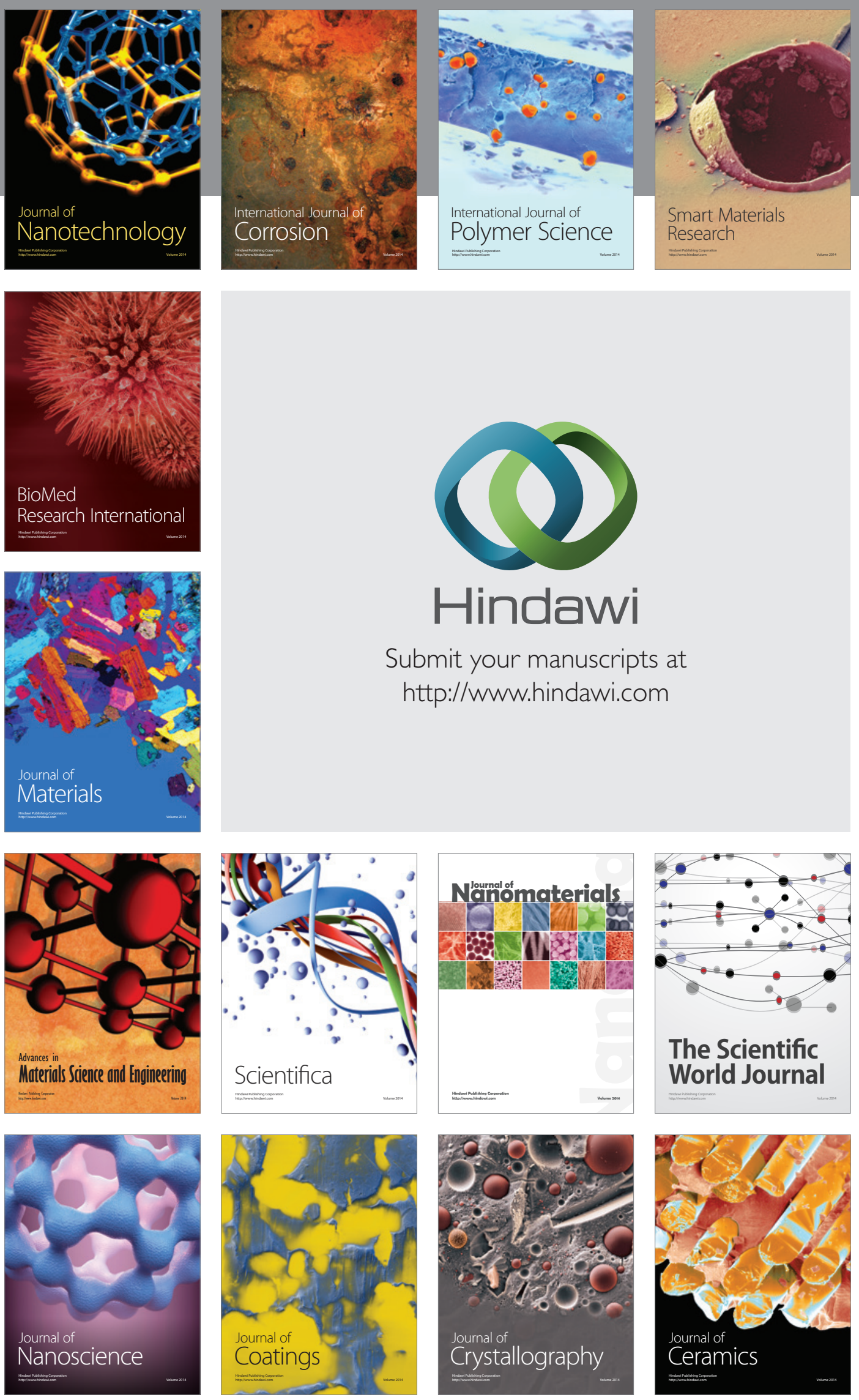

The Scientific World Journal

Submit your manuscripts at

http://www.hindawi.com

\section{World Journal}

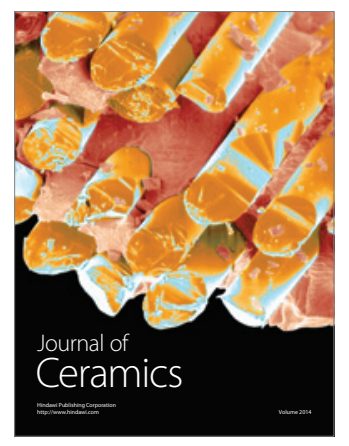

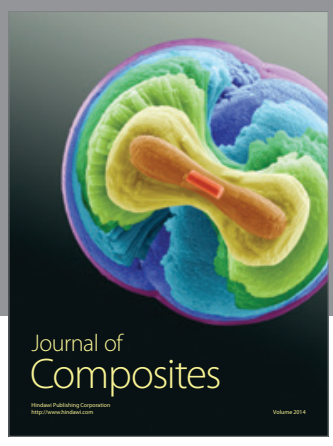
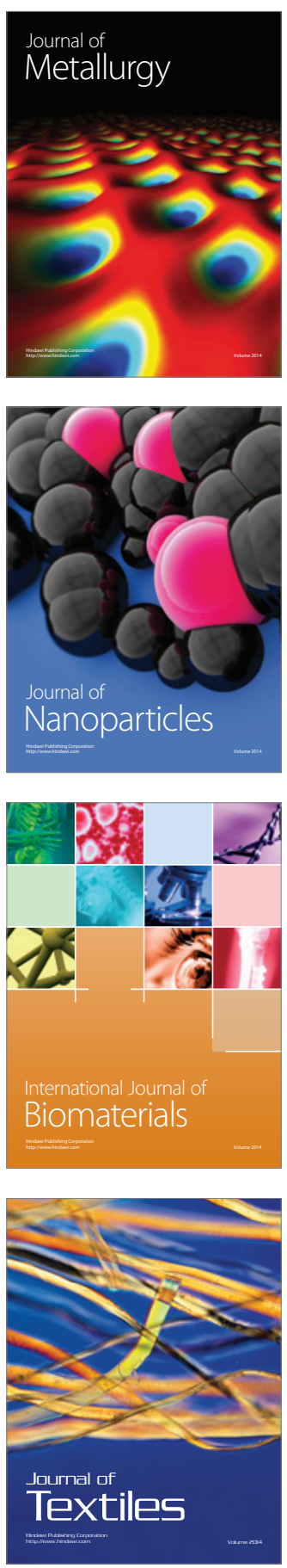